\title{
National survey of practices to prevent health care-associated infections in Thailand: The role of prevention bundles
}

\author{
Anucha Apisarnthanarak MD a,*, David Ratz MS b ${ }^{\text {, }}$ M. Todd Greene PhD, MPH b,c, \\ Thana Khawcharoenporn MD, MSc a , David J. Weber MD, MPH ${ }^{\mathrm{d}}$, \\ Sanjay Saint MD, MPH ${ }^{\mathrm{b}, \mathrm{c}}$ \\ a Department of Medicine, Thammasat University, Pathumthani, Thailand \\ b Center for Clinical Management Research, Veterans Affairs Ann Arbor Healthcare System, Ann Arbor, M \\ ${ }^{\mathrm{c}}$ Division of General Medicine, University of Michigan, Ann Arbor, MI \\ d Schools of Medicine and Public Health, University of North Carolina, Chapel Hill, NC
}

Key Words:

Cross-contamination

Patient safety

Catheter-associated urinary tract infection

Bloodstream infection

Ventilator-associated pneumonia
Background: We evaluated the practices used in Thai hospitals to prevent catheter-associated urinary tract infection (CAUTI), central line-associated bloodstream infection (CLABSI), and ventilator-associated pneumonia (VAP).

Methods: From January 1, 2014-November 30, 2014, we surveyed all Thai hospitals with an intensive care unit and at least 250 beds. The use of prevention practices for CAUTI, CLABSI, and VAP was assessed. High compliance $(\geq 75 \%)$ with all components of the CLABSI and VAP prevention bundles were determined. CAUTI, CLABSI, and VAP infection rates before and after implementing infection control practices are reported. Multivariable regression was used to examine associations between infection prevention bundle compliance and infection rate changes.

Results: Out of 245 eligible hospitals, 212 (86.5\%) responded. A total of 120 (56.6\%) and 115 hospitals (54.2\%) reported $\geq 75 \%$ compliance for all components of the CLABSI and VAP prevention bundles, respectively, and 91 hospitals (42.9\%) reported using $\geq 4$ recommended CAUTI-prevention practices. High compliance with all of the CLABSI and VAP bundle components was associated with significant infec-tion rate reductions (CLABSI, 38.3\%; $P<.001$; VAP, 32.0\%; $P<.001$ ). Hospitals regularly using $\geq 4$ CAUTIprevention practices did not have greater reductions in CAUTI $(0.02 \% ; P=.99)$.

Conclusions: Compliance with practices to prevent hospital infections was suboptimal. Policies and interventions promoting bundled approaches may help reduce hospital infections for Thai hospitals.
In Southeast Asia, the risk of health care-associated infection (HAI) is higher than in developed countries with infection related to invasive medical devices remaining a major challenge. ${ }^{1}$ Several factors are associated with a high incidence of hospital infection in Southeast Asian countries, including limited resources in most hospitals, lack of support for infection control from administration, inadequate nurse to patient ratios, limited spending on HAI prevention, as well as limited national policies to help prevent infection. ${ }^{1-3}$

\footnotetext{
* Address correspondence to Anucha Apisarnthanarak, MD, Division of Infectious Diseases, Thammasat University Hospital, Pratumthani 12120, Thailand.

E-mail address: anapisarn@yahoo.com (A. Apisarnthanarak).

This study was supported by the National Research University Project of the Thailand Office of Higher Education Commission to AA.

Conflicts of interest: None to report.
}

The need to increase awareness of HAI prevention has been recognized among Thai health care professionals since $2007 .{ }^{1}$ Commitment to the World Health Organization Patient Safety Campaign has led to several hospital initiatives to prevent hospital infections throughout the country..$^{4-8}$ Despite these efforts, there are potential gaps in translating the existing knowledge of implementing bundles of care for HAI prevention into clinical practice in a majority of Thai hospitals. In 2010, we conducted an initial national survey of practices to prevent HAI in hospitals across Thailand. ${ }^{9}$ Although many infection-prevention practices for HAI were used infrequently, hospitals participation in an HAI collaborative network was associated with more frequent use of certain recommended HAIprevention practices from the previous Thai national survey. ${ }^{9}$ To understand the current practices used to prevent HAI and develop further insights into the potential benefits of bundled prevention approaches, we conducted a follow-up national survey. We were 
especially curious to examine the extent to which compliance with prevention bundles influence common infections.

\section{METHODS}

\section{Survey instrument}

From January 1, 2014-November 30, 2014, we surveyed all hospitals in Thailand that had an intensive care unit and at least 250 hospitals beds $(\mathrm{N}=245)$. The list of included hospitals was obtained from the Thai Ministry of Public Health. The survey instrument was first developed by Krein, et $\mathrm{ll}^{10,11}$ and has undergone multiple recent revisions. The survey was translated into Thai by an experienced hospital epidemiologist (AA). The survey assessed general hospital, personnel, and infection control program characteristics, as well as the practices used by Thai hospitals to prevent common hospital infections, including catheter-associated urinary tract infection (CAUTI), central line-associated bloodstream infection (CLABSI), and ventilator-associated pneumonia (VAP).

The lead infection preventionist (IP) for each hospital was interviewed to determine various hospital characteristics, as well as the frequency of numerous infection-prevention practices being used. IPs were asked about how often their hospital used specific prevention practices ( 1 = never to 5 = always) for CAUTI, CLABSI, and VAP. Responses of 4 or 5 were coded as "regular use" and the dichotomized variables were used in all analyses. The level of compliance with bundled approaches for CLABSI and VAP prevention were also assessed. Hospitals were asked how often they complied with the given bundled practices $(1=100 \%$ to $6=$ no monitoring of compliance). Responses of 1 or 2 (75\%-100\%) were coded as frequent compliance for all analyses. The CLABSI bundle consisted of hand hygiene, maximum sterile barrier precautions, use of chlorhexidine gluconate for antisepsis of the insertion site, choosing optimal site for line insertion, and daily review of line necessity. The VAP bundle consisted of hand hygiene, semirecumbent positioning of the patient, avoidance of frequent ventilator circuit changes, use of antimicrobial mouth rinse, feeding content check, and cross-contamination prevention. Because a specific CAUTI bundle was not yet promulgated in Thailand, we examined the influence of regularly using at least 4 of 6 evidence-based CAUTI-prevention practices: portable bladder ultrasound, urinary catheter reminder/ stop order or nurse-initiated urinary catheter discontinuation, condom catheters in men, aseptic technique during indwelling urethral catheter insertion and maintenance, intermittent catheterization, and maintaining a closed urinary catheter system. In addition to prevention practices, IPs were also asked about their specific hospital HAI infection rates. Data on hospital CAUTI, CLABSI, and VAP infection rates both 12 months before and 12 months after implementing infection control practices to prevent each infection type were collected based on the existing infection control data maintained at each hospital.

In-person interviews were administered by research nurses who used the survey instrument to interview each of the lead IPs. Three training sessions were held to instruct the 5 research nurses on the survey and data collection procedures. The survey instrument was pilot tested in 10 hospitals to ensure the validity, reliability, and acceptability of the survey results by 5 research nurses; $100 \%$ agreement in the responses captured was observed in the pilot test. This study was approved by the Institutional Review Board of the Faculty of Medicine, Thammasat University.

\section{Statistical analysis}

Descriptive statistics were calculated for all relevant survey questions. Multivariable logistic regression was used to determine significant associations between hospital characteristics and regular use of each infection-prevention practice. The hospital characteristics considered were type of ownership, number of acute care beds, affiliation with medical school, whether or not the hospital has hospitalists, involvement in a collaborative to reduce HAI, support for an infection control program from leadership, whether or not the hospital has a hospital epidemiologist, total full-time equivalent of all IPs, and whether the lead IP is certified in infection control. Additionally, whether or not the hospital has an infectious diseases specialist, environmental cleaning service, facilities maintenance department, or microbiology lab were also included if they were statistically significant in a bivariable model. One CAUTI practice (ie, portable bladder ultrasound) was excluded from the logistic regression analyses due to low use ( $<5 \%$ regular use).

Linear regression models were used to look at relative reductions in HAI rates. The primary exposure variables were regular use of 4 of 6 recommended CAUTI practices (for the CAUTI model), at least $75 \%$ compliance with practicing all components of the bundle for CLABSI (for the CLABSI model), and at least 75\% compliance with practicing all components of the bundle for VAP (for the VAP model). In addition to the covariates mentioned above in the logistic regression analyses, the relative reduction models were additionally adjusted for whether or not the hospital has an established surveillance system for monitoring infection rates. All statistical analyses were performed using SAS version 9.4 (SAS Institute Inc, Cary, NC).

\section{RESULTS}

Of the 245 eligible hospitals, 212 (86.5\%) responded to our survey. General and infection-specific characteristics of the hospitals are in Table 1 . Nearly $97 \%$ of hospitals reported having hospitalists, 94.8\% have a microbiology lab, and $92.5 \%$ have a lead IP who is certified in infection control. Approximately half of all hospitals were affiliated with a medical school (52.4\%), have infectious disease specialists (50.9\%), and are involved in a collaborative to reduce HAIs (49.5\%). For CAUTI, more than $90 \%$ of responding hospitals have a system to monitor which patients have a urinary catheter placed (92.9\%), routinely monitor duration/discontinuation of urinary catheters (90.6\%), have an established surveillance system for monitoring CAUTI rates (96.7\%), and report CAUTI rates to direct care providers (93.9\%). Similarly, for CLABSI and VAP, the vast majority of hospitals have an established surveillance system for monitoring rates ( $91.5 \%$ for both) and report rates to direct care providers ( $87.7 \%$ and $90.1 \%$, respectively).

The percentage of hospitals that regularly use key prevention practices for CAUTI, CLABSI, and VAP are listed in Figure 1. The most frequently used CAUTI prevention practices among Thai hospitals were using a closed urinary catheter system (90.1\% regular use) and aseptic technique during indwelling urethral catheter insertion and maintenance (89.6\%). For CLABSI, chlorhexidine gluconate for antisepsis of the insertion site (73.6\%) and maximum sterile barrier precautions during catheter insertion (63.2\%) were the most common prevention practices. For VAP, semirecumbent positioning (86.8\%) and antimicrobial mouth rinse (78.3\%) were most frequently used. A total of 120 (56.6\%) hospitals reported $\geq 75 \%$ compliance rate for all components of the CLABSI prevention bundle and 115 hospitals $(54.2 \%$ ) reported $\geq 75 \%$ compliance rate for all components of the VAP prevention bundle. Although a specific CAUTI prevention bundle was not yet recommended, 91 hospitals (42.9\%) reported using $\geq 4$ CAUTI-prevention practices.

Significant associations between hospital characteristics and regular use of various HAI prevention practices use can be found in Table 2. Hospitals with strong leadership support for infection control were more likely to use semirecumbent positioning of the patient, antimicrobial mouth rinse, and oscillating/kinetic beds for 
Table 1

General hospital characteristics $(\mathrm{N}=212)$

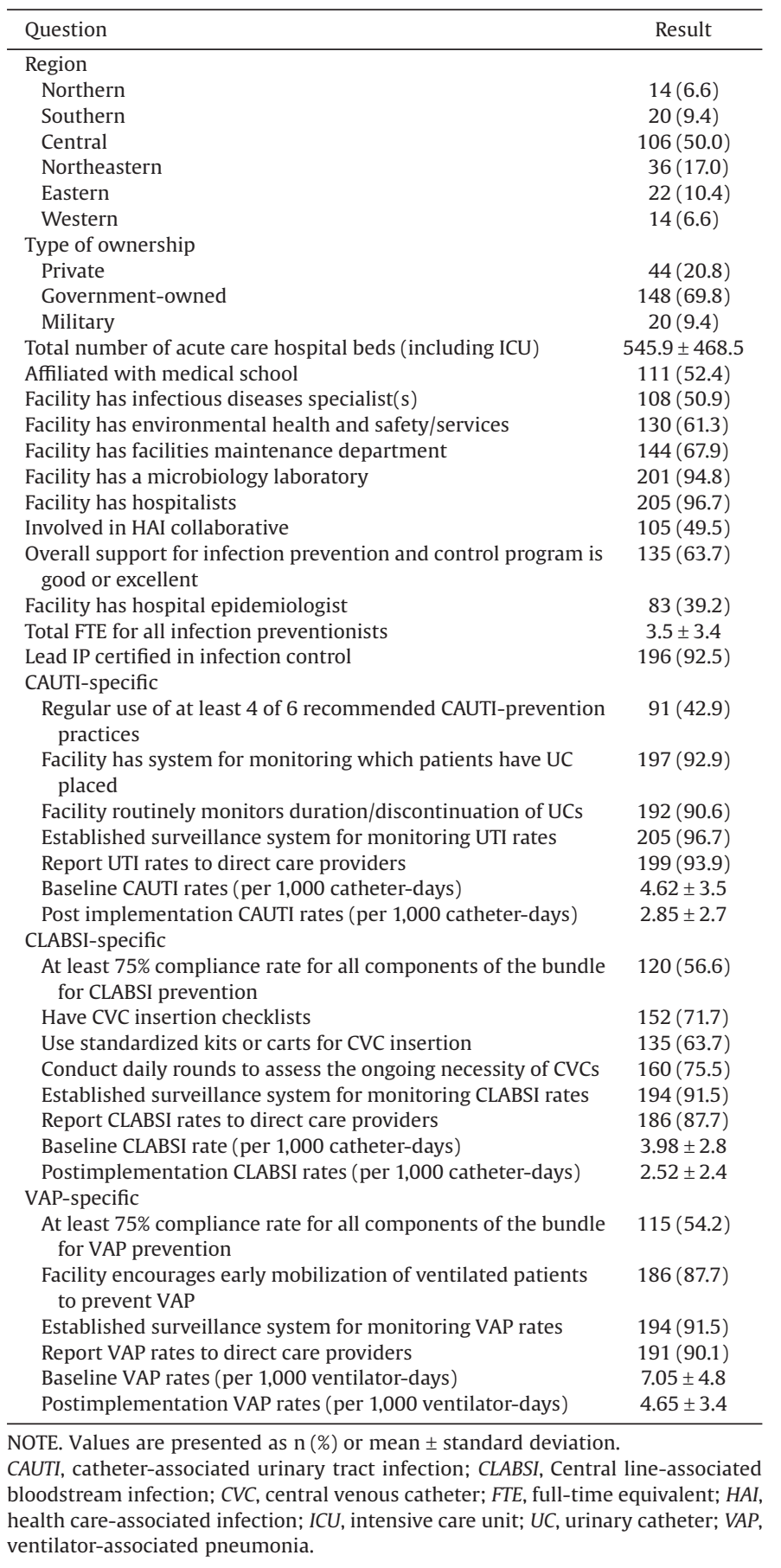

VAP prevention, as well as using chlorhexidine gluconate for insertion site antisepsis and avoiding the femoral site for line insertions for CLABSI prevention. Hospitals that were involved in an HAI collaborative were more likely to regularly use antimicrobial dressing with chlorhexidine and avoid the femoral site for line insertions for CLABSI prevention, as well as semirecumbent positioning and antimicrobial mouth rinse for VAP prevention. Hospitals that have a hospital epidemiologist were more likely to regularly use urinary catheter reminder or stop-order or nurse-initiated catheter discontinuation, antimicrobial catheters, and condom catheters in men for CAUTI prevention. Having a lead IP certified in infection control was associated with more regularly using antimicrobial dressing with chlorhexidine for CLABSI prevention, as well as semirecumbent positioning of the patient and antimicrobial mouth rinse for VAP prevention.

Multivariable linear regression results for HAI rate relative reductions can be found in Table 3. Participating in an HAI collaborative was associated with significant decreases in CAUTI, CLABSI, and VAP rates $(13.4 \%, 19.2 \%$, and $23.3 \%$ reductions, respectively). The presence of facilities maintenance department was associated with significant decreases in CAUTI, CLABSI, and VAP rates $(18.7 \%, 13.7 \%$, and $15.0 \%$ reductions, respectively). High compliance $(\geq 75 \%)$ with all of the components of the CLABSI and VAP bundles was also associated with significant decreases in their respective rates (CLABSI, $38.3 \%$ reduction and VAP, 32.0\% reduction). Regular use of 4 of 6 recommended CAUTI practices was not associated with decreased CAUTI rates $(0.02 \%$ reduction; $P=.99)$.

\section{DISCUSSION}

Several key findings emerged from our national Thai study. First, despite having committed to the World Health Organization Patient Safety Campaign since 2007, practices to prevent major hospital infections are still practiced infrequently in Thailand. Second, despite suboptimal use of certain infection-prevention practices, we did identify several key hospital factors associated with increased use of numerous HAI-prevention practices. Third, our study supports the role of HAI care bundles and the need to have a high compliance level for bundle use to achieve reduction in HAIs in this middleincome country.

National surveys of practices to prevent HAI-using similar instruments-have previously been conducted in several countries, including the United States, Thailand, and Japan. ${ }^{9,12-16}$ Compared with the United States, practices to prevent HAI have generally been used less frequently in Asian countries. ${ }^{9,16}$ Despite this, the presence of a strong organizational safety culture has been shown to improve HAI prevention efforts in Thailand and Japan ${ }^{9,16}$ and participating in an HAI collaborative network has a strong association with improved HAI prevention in Thailand. ${ }^{9}$ Similarly, in this study we found that being involved in a collaborative and having the support of leadership are important factors associated with higher use of infection-prevention practices. Additionally, we also found that having a hospital epidemiologist on staff was associated with more regular use of several key infection-prevention practices. This association may be in part explained by the curriculum created by Thai government to provide formal training for postgraduate physicians in infection prevention to become a hospital epidemiologist. This finding highlights an opportunity for countries in Asia to provide formal training in hospital epidemiology as part of strategic infectionprevention plans.

Bundles of care are now encouraged for use to prevent infections throughout the world. ${ }^{17-20}$ Previous studies in the United States have shown the positive influence that complying with any 1 of 3 CLABSI bundle elements or complying with 2 VAP bundle components had on reductions in CLABSI and VAP rates, respectively. ${ }^{21,22}$ In this study, we found that achieving high compliance $(\geq 75 \%)$ with all components of prevention care bundles was associated with reduction in CLABSI and VAP rates. Our findings thus suggest that strict adherence to all HAI bundle components may hold promise for further reductions in HAI outcomes in Thailand. Because there was no existing CAUTI bundle in Thailand at the time of survey, the influence of a CAUTI bundle as well as the compliance level needed to achieve reduction in CAUTI rates cannot be determined. However, hospitals that more frequently used a combination of numerous CAUTI prevention practices did not demonstrate greater reductions in their CAUTI rates, compared with hospitals using fewer practices. 


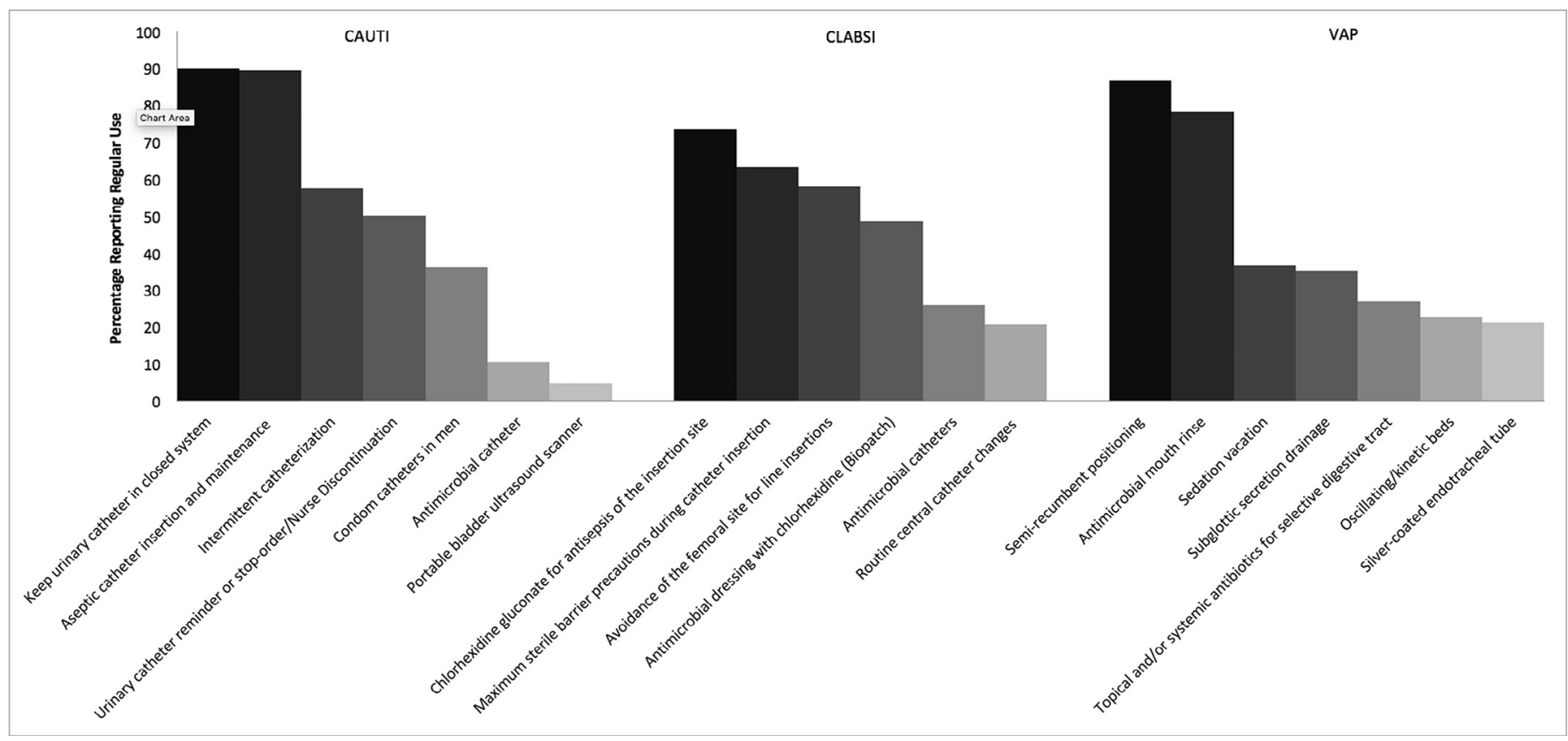

Fig 1. Percentage of reporting hospitals regularly using select health care-associated infection practices.

Table 2

Multivariable logistic regression results: Factors significantly associated with more frequent use of prevention practices

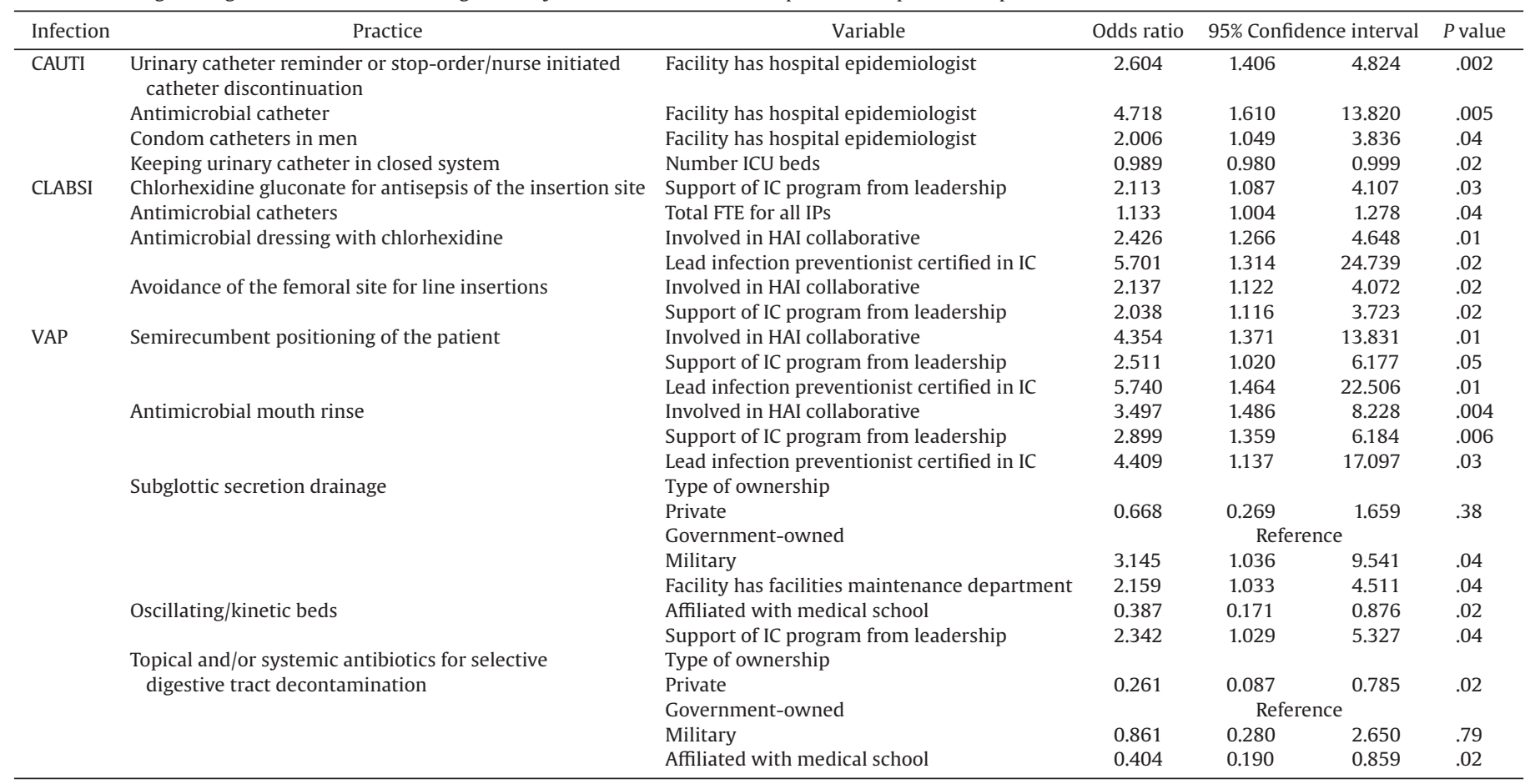

CAUTI, catheter-associated urinary tract infection; CLABSI, central line-associated bloodstream infection; IC, infection control; ICU, intensive care unit; FTE, Full-time equivalent; VAP, ventilator-associated pneumonia.

Several limitations of our study merit discussion. First, because the response rate was $<100 \%$, our results are susceptible to nonresponse bias. Although we achieved a very high response rate, our findings may not be generalizable to all hospitals in Thailand. Second, because we relied on self-reported data from lead IPs to determine the frequency of the various infection-prevention practices being used, there is a potential for respondent bias. Third, hospital infection rates at 2 time points (aggregate 12 months before and 12 months after interventions were introduced) were based on reported estimates from the lead IP at each hospital. Absent longitudinal infection rate data, reported rates in our study are prone to response bias. Fourth, several infection-prevention practices were used infrequently, limiting our ability to investigate their influence on reducing HAI rates directly. Fifth, because the compliance 
Table 3

Multivariable regression results: Factors significantly associated with a relative change in infection rates

\begin{tabular}{|c|c|c|c|c|c|}
\hline \multirow{2}{*}{$\begin{array}{l}\text { Infection } \\
\text { CAUTI }\end{array}$} & \multirow{2}{*}{$\begin{array}{c}\text { Variable } \\
\text { Facility have has facilities maintenance department }\end{array}$} & \multirow{2}{*}{$\frac{\text { Estimate }}{-0.187}$} & \multicolumn{2}{|c|}{ 95\% Confidence interval } & \multirow{2}{*}{$\frac{P \text { value }}{.002}$} \\
\hline & & & -0.305 & -0.069 & \\
\hline & Involved in HAI collaborative & -0.134 & -0.247 & -0.020 & .02 \\
\hline & Total FTE for all infection preventionists & -0.027 & -0.046 & -0.008 & .01 \\
\hline & Regular use of 4 of 6 recommended CAUTI practices & -0.0002 & -0.114 & 0.114 & .99 \\
\hline \multirow[t]{5}{*}{ CLABSI } & Facility have has facilities maintenance department & -0.137 & -0.269 & -0.004 & .04 \\
\hline & Involved in HAI collaborative & -0.192 & -0.321 & -0.062 & .004 \\
\hline & Facility has hospital epidemiologist & 0.144 & 0.021 & 0.267 & .02 \\
\hline & Lead infection preventionist certified in IC & -0.261 & -0.487 & -0.034 & .02 \\
\hline & Compliance rate in your hospital to practice all of the components in the bundle for CLABSI ${ }^{* \dagger}$ & -0.383 & -0.513 & -0.254 & $<.0001$ \\
\hline \multirow[t]{8}{*}{ VAP } & Type of ownership & & & & \\
\hline & Private & -0.123 & -0.258 & 0.013 & .08 \\
\hline & Government-owned & \multicolumn{4}{|c|}{ Reference } \\
\hline & Military & 0.157 & -0.021 & 0.336 & .08 \\
\hline & Facility have has facilities maintenance department & -0.150 & -0.259 & -0.040 & .01 \\
\hline & Involved in HAI collaborative & -0.233 & -0.341 & -0.125 & $<.0001$ \\
\hline & Facility has hospital epidemiologist & 0.146 & 0.043 & 0.250 & .01 \\
\hline & Compliance rate in your hospital to practice all of the components in the bundle for VAP*广 & -0.320 & -0.428 & -0.212 & $<.0001$ \\
\hline
\end{tabular}

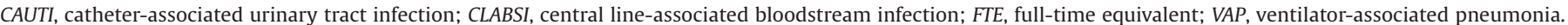

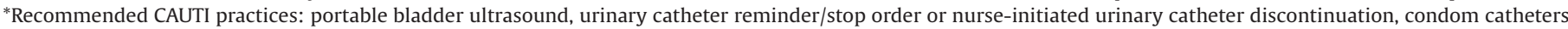

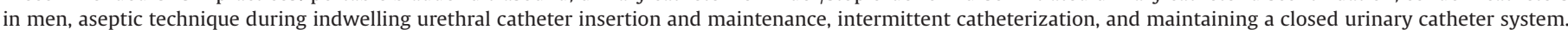

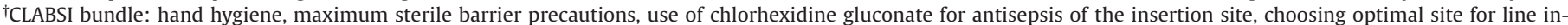
sertion, and daily review of line necessity.

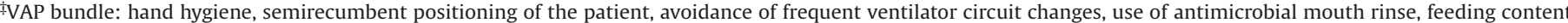
check, and cross-contamination prevention.

rates with the CLABSI and VAP care bundles were reported by the lead IPs and derived from hospital-specific infection control data, they may not reflect actual compliance. Finally, we did not have access to (and thus could not adjust for) patient-level or hospital case-mix data. As such, our regression estimates could be biased because of unmeasured confounding, and our results can only be interpreted as providing evidence for associations rather than causal mechanisms.

\section{CONCLUSIONS}

Our study identified several key strategies on which Thai hospitals engaged in infection-prevention efforts should focus. Focusing on participating in infection-prevention collaboratives, garnering strong leadership support for infection-prevention efforts, having a hospital epidemiologist on staff to champion the resources and buy-in necessary for successful infection control, and promoting certification in infection control among lead IPs may help in achieving and sustaining reductions in HAI rates. Although such an infrastructure shows promise, several gaps still exist between the actual use of HAI-prevention practices and the national expectations of patient safety. Importantly, our study highlights the importance of promoting bundled prevention approaches to help reduce hospital infections in this developing country. The promotion of best infection-prevention practices based on expert consensus, policies for bundled infection-prevention approaches, as well as dedicated efforts to increase health care workers' compliance with such approaches, should be considered as part of focused collaborative national efforts to reduce HAI in Thailand.

\section{References}

1. Ling ML, Apisarnthanarak A, Madriaga G. The burden of healthcare associated infections in Southeast Asia: a systematic literature review and meta-analysis. Clin Infect Dis 2015;60:1690-9.

2. Apisarnthanarak A, Khawcharoenporn T, Greene MT, Kennedy E, Krein S, Saint S. National survey of Thai Infection Prevention in the era of patient safety. Am J Infect Control 2013;41:362-4.

3. Rosenthal VD, Lynch P, Jarvis WR, Khader IA, Richtmann R, Jaballah NB, et al. Socioeconomic impact on device-associated infections in limited-resource neonatal intensive care units: findings of the INICC. Infection 2011;39:439-50.
4. Korbkitjaroen M, Vaithayapichet S, Kachintorn K, Jintanothaitavorn D, Wiruchkul $\mathrm{N}$, Thamlikitkul V. Effectiveness of comprehensive implementation of individualized bundling infection control measures for prevention of health care-associated infections in general medical wards. Am J Infect Control 2011;39:471-6.

5. Rattanaumpawan P, Teeratorn N, Thamlikitkul VA. Cluster-randomized controlled trial of the catheter reminder and evaluation program. Infect Control Hosp Epidemiol 2016;37:201-3.

6. Apisarnthanarak A, Thongphubeth K, Sirinvaravong S, Kitkangvan D, Yuekyen C, Warachan B, et al. Effectiveness of multifaceted hospitalwide quality improvement programs featuring an intervention to remove unnecessary urinary catheters at a tertiary care center in Thailand. Infect Control Hosp Epidmiol 2007:28:791-8.

7. Unahalekhaka A, Jamulitrat S, Chongsuvivatwong V, Øvretveit J. Using a collaborative to reduce ventilator-associated pneumonia in Thailand. Jt Comm J Qual Patient Saf 2007;33:387-94.

8. Apisarnthanarak A, Bangsong R, Saelao A, Pothirat T, Rutjanawech S, Khawcharoenporn T, et al. Assessment of the 2007 Thai commitment to the Global Patient Safety Campaign. Am J Infect Control 2014;42:690-1.

9. Apisarnthanarak A, Greene MT, Kennedy EH, Khawcharoenporn T, Krein S, Saint S. National survey of practices to prevent healthcare-associated infections in Thailand: the role of safety culture and collaboratives. Infect Control Hosp Epidemiol 2012:33:711-7.

10. Krein SL, Olmsted RN, Hofer TP, Kowalski C, Forman J, Banaszak-Holl J, et al. Translating infection prevention evidence into practice using quantitative and qualitative research. Am J Infect Control 2006;34:507-12.

11. Krein SL, Fowler KE, Ratz D, Meddings J, Saint S. Preventing device-associated infections in US hospitals: national surveys from 2005 to 2013. BMJ Qual Saf 2015;24:385-92.

12. Krein SL, Hofer TP, Kowalski CP, Olmsted RN, Kauffman CA, Forman JH, et al. Use of central venous catheter-associated bloodstream infections prevention practices by US hospital. Mayo Clin Proc 2007;82:672-8.

13. Krein SL, Kowalski CP, Damschroder L, Forman J, Kaufman SR, Saint S. Prevention ventilator-associated pneumonia in the United States: a multi-center mixedmethod study. Infect Control Hosp Epidemiol 2008;29:933-40.

14. Greene MT, Kiyoshi-Teo $H$, Reichert $H$, Krein S, Saint S. Urinary catheter indication in the United States: results from a national survey from acute care hospitals. Infect Control Hosp Epidemiol 2014;35(Suppl 3):S96-8.

15. Krein SL, Fowler KE, Ratz D, Meddings J, Saint S. Preventing device associated infections in US hospital: national survey from 2005-2013. BMJ Qual Saf 2015;24:385-92.

16. Sakamoto F, Sakihama T, Saint S, Greene MT, Ratz D, Tokuda Y. Health careassociated infection prevention in Japan: the role of safety culture. Am J Infect Control 2014;42:888-93

17. Yokoe DS, Anderson DJ, Berenholtz SM, Calfee DP, Dubberke ER, Ellingson KD, et al. A compendium of strategies to prevent healthcare-associated infections in acute care hospitals: 2014 updates. Infect Control Hosp Epidemiol 2014;35(Suppl 2):S21-31.

18. Marschall J, Mermel LA, Fakih M, Hadaway L, Kallen A, O'Grady NP, et al. Strategies to prevent central line-associated bloodstream infections in acute care hospitals: 2014 update. Infect Control Hosp Epidemiol 2014;35:753-71. 
19. Lo E, Nicolle LE, Coffin SE, Gould C, Maragakis LL, Meddings J, et al. Strategies to prevent catheter-associated urinary tract infections in acute care hospitals: 2014 update. Infect Control Hosp Epidemiol 2014;35:464-79.

20. Coffin SE, Klompas M, Classen D, Arias KM, Podgorny K, Anderson DJ, et al. Strategies to prevent ventilator-associated pneumonia in acute care hospitals. Infect Control Hosp Epidemiol 2008;29(Suppl 1):S31-40.
21. Pogorzelska M, Stone PW, Furuya EY, Perencevich EN, Larson EL, Goldmann D, et al. Impact of the ventilator bundle on ventilator-associated pneumonia in intensive care unit. Int J Qual Health Care 2011;23:538-44.

22. Furuya EY, Dick A, Perencevich EN, Pogorzelska M, Goldmann D, Stone PW. Central line bundle implementation in US intensive care units and impact on bloodstream infections. PLoS 2011;6:e15452. 